\title{
Selection of grandparental combinations as a procedure designed to make use of dominance genetic effects
}

\author{
Miguel A. Toro \\ CIT-INIA, Area de Mejora Genética Animal, Carretera La Coruña Km.7, \\ 28040 Madrid, Spain
}

(Received 2 March 1998; accepted 27 May 1998)

\begin{abstract}
A general procedure called selection of grandparental combinations (SGPC) is presented, which allows one to use dominance genetic effects. The method assumes that there are two types of matings: either to breed the population or to obtain commercial animals. The idea is to select grandparental combinations such that the overall genetic merit of future grandoffspring which constitute the commercial animals is maximized. Two small computer simulated examples are analysed assuming either a infinitesimal genetic model or that QTL controlling the trait are known. (C) Inra/Elsevier, Paris

selection of grandparental combinations / dominance variance / mating strategies
\end{abstract}

Résumé - Sélection de combinaisons de grands-parents comme une procédure pour utiliser les effets de dominance génétique. On présente une procédure générale appelée sélection de combinaisons de grands-parents (SGPC), qui permet l'utilisation des effets de dominance génétique. La méthode suppose qu'il y a deux types d'accouplements, l'un pour propager la population, l'autre pour l'obtention des animaux commerciaux. L'objectif est de sélectionner les combinaisons de grandsparents de telle façon que le mérite génétique global des futurs petit-fils, qui constituent les animaux commerciaux, soit maximisé. Deux petits exemples de simulation sur ordinateur sont analysés, l'un supposant le modèle génétique infinitésimal, et l'autre introduisant des QTL qui contrôlent le caractère. (C) Inra/Elsevier, Paris

sélection de combinaisons de grands-parents / variance de dominance / stratégies d'accouplement

E-mail: toro@inia.es 


\section{INTRODUCTION}

Breeding programmes for economically important traits are based on selecting as parents for the next generation the individuals with highest genetic merit estimated by mixed model methodology. However, in the near future, molecular information will be integrated into mixed models to achieve the maximum improvement. If the loci affecting a quantitative trait (QTL), were known, it would be possible to directly select specific alleles, or if genetic markers linked to QTL were detected, they could also be used in marker-assisted selection.

In any case, profit from dominance genetic effects in breeding programmes can only be obtained when final commercial animals are the product of matings other than those involved in the maintenance of the breeding population. In a large number of domestic species, the final product is the result of twoway, three-way or rotational crossbreeding among breeds or strains that are maintained separately. In this context, selection is independently carried out in each parental population and, in addition, the value of the cross may increase as a result of heterosis. An exception to this practice is the reciprocal-recurrent selection scheme (RRS) [1], whose merits relative to pure-line selection (PLS) have been reviewed by Wei and van der Steen [14].

Several authors have suggested that although selection should be carried out on estimated additive breeding values, animals used for commercial production should be the product of planned matings which maximize the overall (additive plus dominance effects) genetic merit of the offspring [4, 8]. More recently, Toro [12] claimed that dominance genetic variance can also be exploited in a closed population, as long as different mating systems are applied for providing breeding commercial animals.

In this note, we present a more general procedure, i.e. selection of grandparental combinations (SGPC), as proposed by Toro [13], which is not restricted to the progeny test scheme. Moreover, SPGC benefits from the use of mixed model methodology, which is considered as the method of choice for genetic evaluation in animal breeding.

\section{THEORY}

The methodology suggested by Toro [12] basically consists in making two different types of matings in the framework of a progeny test scheme: a) minimum coancestry matings to obtain commercial animals that will also be used for estimating breeding values of nucleus animals; and b) maximum coancestry matings from which the population will be propagated. Simulation results showed that the superiority of this new method over the standard progeny test depends on the genetic architecture of the trait and that it is especially effective if there is overdominance or if there are unfavourable recessive alleles at low frequencies.

This method has two main limitations. First, it is not optimized with respect to the proportion of matings among relatives both to obtain commercial animals and to propagate the population. Second, it is limited to a progeny test breeding scheme. The method proposed in the present paper, called selection of grandparental combinations (SGPC), is not restricted to a progeny test scheme 
and it is aimed at optimizing the proportion of matings among relatives in both the commercial and the breeding population.

Consider, for the sake of simplicity, a population of three males $(1,2,3)$ and three females $(4,5,6)$. The objective is to select two mating pairs to propagate the population from the nine potential ones shown in table $I$. At some future time, the commercial animals will be the grandoffspring of the individuals considered and, therefore, the progeny of one of the 18 potential grandparental combinations, assuming that each male can only be mated with one female (table I). Thus, we should select the combination which maximizes the expected value of the overall genetic merit of the future commercial animals. If, for example, the expected genetic merit of the grandoffspring of $(1 \times 4) \times(2 \times 6)$ is the highest, we should select mating pairs $1 \times 4$ and $2 \times 6$ for the propagation of the population. The genetic values of these expected grandoffspring could be predicted using mixed model methodology including dominance and inbreeding genetic effects. An intuitive interpretation would be as follows. If, for example, a trait is controlled by a biallelic locus showing overdominance, the best grandparental combination for obtaining future commercial animals would be $(\mathrm{AA} \times \mathrm{AA}) \times(\mathrm{aa} \times \mathrm{aa})$, because it produces heterozygous Aa grandoffspring. Obviously, mating pairs AA $\times$ AA and aa $\times$ aa should be chosen to propagate the population.

\section{SIMULATION}

Because of the rather intuitive justification of the method given above, the performance of the newly proposed method was checked by computer simulation assuming either an infinitesimal model or a model based on known genetic loci.

\subsection{Breeding scheme}

Selection was carried out over six generations following closely the scheme presented in table $I$ but considering a population of 32 candidates (16 males and 16 females) instead of six candidates (three males and three females). Each generation, four combinations of potential grandparents (eight mating pairs) were selected according to the predicted genetic merit of their grandoffspring. Although the most appropriate technique for selecting the best grandparental combinations would be linear programming, a simpler and computationally faster strategy that sequentially chooses the best available combinations was used [9]. As indicated by this author, this strategy is generally close to optimal. The new method was compared with a standard selection method in which potential grandparents were selected according to their average predicted additive genetic value. The number of replicates was 200 for the infinitesimal genetic model and 100 for the finite loci model.

\subsection{Infinitesimal genetic model}

The total phenotypic effect of an individual, $y$, was simulated as

$$
y=a+d+b F+e
$$


Table I. Scheme of the new method: selection of grandparental combinations (SGPC).

\begin{tabular}{|c|c|c|c|}
\hline Animals & $\begin{array}{l}\circlearrowleft \\
q\end{array}$ & $\begin{array}{l}1 \\
4\end{array}$ & $\begin{array}{l}2 \\
5\end{array}$ \\
\hline Potential mating pairs & & & $\begin{array}{l}1 \times 4 \\
1 \times 5 \\
1 \times 6 \\
2 \times 4 \\
2 \times 5 \\
2 \times 6 \\
3 \times 4 \\
3 \times 5 \\
3 \times 6\end{array}$ \\
\hline Potential future matings for the commercial population & & $\begin{array}{l}(1 \\
(1 \\
(1 \\
(1 \\
(1 \\
(1 \\
(1 \\
(1 \\
(1 \\
(1 \\
(1 \\
(1 \\
(2 \\
(2 \\
(2 \\
(2 \\
(2 \\
(2\end{array}$ & $\begin{array}{l}\times(2 \times 5) \\
\times(2 \times 6) \\
\times(3 \times 5) \\
\times(3 \times 6) \\
\times(2 \times 4) \\
\times(2 \times 6) \\
\times(3 \times 4) \\
\times(3 \times 6) \\
\times(2 \times 4) \\
\times(2 \times 5) \\
\times(3 \times 4) \\
\times(3 \times 5) \\
\times(3 \times 5) \\
\times(3 \times 6) \\
\times(3 \times 4) \\
\times(3 \times 6) \\
\times(3 \times 4) \\
\times(3 \times 5)\end{array}$ \\
\hline
\end{tabular}

where $a$ is the additive value, $b$ and $F$ are the inbreeding depression and the coefficient of inbreeding of the individual, $d$ the dominance effect and $e$ an environmental random deviate. The dominance effect, ignoring inbreeding, was simulated as its sire $\times$ dam combination effect plus mendelian sampling [7]

$$
d=f_{S, D}+\delta
$$

where $f_{S, D}$ represents the average dominance effect of many hypothetical fullsibs produced by the individual's sire $S$ and dam $D$, and $\delta$ is the individual's deviation from the sire $\times$ dam subclass effect. Variances are $V\left(f_{S, D}\right)=0.25 V_{D}$ and $V(\delta)=0.75 V_{D}$, where $V_{D}$ is the dominance variance.

Genetic evaluation was carried out using only phenotypic information from breeding individuals in current and previous generations to estimate additive and dominance effects. First, the following statistical model was used

$$
y_{i}^{*}=y_{i}-b F_{i}=a_{i}+d_{i}+e_{i}
$$


where $y_{i}$ is the phenotypic value of animal $i, b$ is the inbreeding depression (assumed to be known), and $a_{i}$ and $d_{i}$ are additive and dominance effects of animal $i$, respectively. Other possible fixed effects such as generation effect were ignored for simplicity.

Now, if $\mathbf{m}$ is the vector of genetic merit $m=a+d$, the BLUP of $\mathbf{m}$ is the solution of equations

$$
\left(\mathbf{Z}^{\prime} \mathbf{Z}+\mathbf{M}^{-1}\right) \mathbf{m}=\mathbf{Z}^{\prime} \mathbf{y}^{*}
$$

where $\mathbf{M}=\left(\mathbf{A} V_{A}+\mathbf{D} V_{D}\right) / V_{E}, V_{E}$ being the environmental variance.

The expected additive plus dominance genetic merit of the grandoffspring of a grandparent combination $(i \times j) \times(k \times l)$ was calculated using [6]

$$
g_{i j k l}=\mathbf{G}_{i j k l} \mathbf{M}^{-1} \mathbf{m}
$$

where $\mathbf{G}_{i j k l}$ is the covariance between the genetic merit of the grandoffspring of the grandparental combination $(i \times j) \times(k \times l)$ and the vector of genetic merits $\mathbf{m}$, computed from the additive and dominance relationship matrices. Finally, the predicted total genetic merit was corrected for the inbreeding depression. The standard procedure is based on a genetic evaluation using the same model (including dominance) as for the proposed method.

Different situations with the same genetic parameters $V_{A}=3.25, V_{D}=6.55$ and $V_{E}=6.55$ but increasing levels of inbreeding depression were considered.

\subsection{Finite loci model}

The trait of interest was simulated as controlled by 100 independent loci with equal effects. Genotypic values at each one were $1, d,-1$ for the allelic combinations $\mathrm{BB}, \mathrm{Bb}$ and $\mathrm{bb}$, respectively. Values of $d=0,0.25,1,-1$ and 1.5 were considered representing different degrees of recessivity of the unfavourable allele. The initial frequency of the $b$ allele was 0.20 .

A two-loci model with epistatic interaction was also tested. The genotypic values are given in table II assuming additive $\times$ additive and diminishing epistasis [2]. Fifty pairs of such loci were simulated with initial frequencies of alleles $\mathrm{b}$ and $\mathrm{c}$ of 0.8 .

Table II. Genotypic values for the epistatic model.

\begin{tabular}{lccccccc}
\hline & \multicolumn{3}{c}{ Additive $\times$ additive } & \multicolumn{3}{c}{ Diminishing } \\
& $\mathrm{BB}$ & $\mathrm{Bb}$ & $\mathrm{bb}$ & & $\mathrm{BB}$ & $\mathrm{Bb}$ & $\mathrm{bb}$ \\
\hline $\mathrm{CC}$ & 4.0 & 1.0 & -2.0 & $\mathrm{CC}$ & 4.0 & 4.0 & 3.0 \\
$\mathrm{Cc}$ & 1.0 & 0.25 & -0.5 & $\mathrm{Cc}$ & 4.0 & 4.0 & 3.0 \\
$\mathrm{cc}$ & -2.0 & -0.5 & 1.0 & $\mathrm{cc}$ & 3.0 & 3.0 & 1.0 \\
\hline
\end{tabular}

In the SGPC method, the expected overall genetic merit of the grandoffspring of a grandparental combination $(i \times j) \times(k \times l)$ was predicted calculating the genetic composition of the grandoffspring from simple mendelian rules. In the standard method, the breeding values of the potential grandparents were also calculated in the same way. 


\section{RESULTS}

\subsection{Infinitesimal genetic model}

The values of the genetic mean of the trait during the first six generations of selection, using the standard procedure and the new method are presented in table III, together with the mean inbreeding coefficient for both the commercial and the breeding populations. Strictly speaking, the performance of the breeding population is an observed value, while the performance of the commercial population is an expected value that will be realized with a one-generation delay.

The cases A, B, C and D in table III refer to different situations with the same genetic variance components but increasing levels of inbreeding depression. This is possible in a genetical infinitesimal model where, unlike the typical biallelic genetic model, inbreeding depression and dominance variance are independent.

As shown in table III the new method achieved the objective of obtaining superior performance of the commercial population in all cases. This superiority was attained by inducing some matings among relatives in the breeding population, in order to profit from dominance. Consequently, the performance of the breeding population was worse with SGPC when inbreeding depression was larger, as in cases $\mathrm{C}$ and $\mathrm{D}$.

Nevertheless, with SGPC, the inbreeding coefficient of commercial animals is automatically adjusted depending on the magnitude of inbreeding depression. In case $\mathrm{A}$, inbreeding depression is not important and therefore, a considerable rate of inbreeding is allowed, whereas in case $\mathrm{C}$, the magnitude of inbreeding depression imposes a stronger restriction. Obviously, in case D, the lower inbreeding in the commercial population is the factor that determines its performance.

In cases A-D, it has been assumed that only the performance of the commercial population is economically valuable, but SGPC could easily accommodate selection for both commercial and breeding population performances. Case $\mathrm{E}$ of table III is the same as case D except that the objective of selection is a combination of the expected genetic merit of the commercial grandoffspring and the expected genetic merit of candidates for selection in the next generation, giving the same weight to both expected values. Although this equal weighting is arbitrary, it highlights the fact that both commercial and breeding population performances could be included. The results indicate that the lower performance of the commercial population is compensated by the superior performance of the breeding population.

\subsection{QTL identified}

Table $I V$ shows that the results with the defined genetic model are similar to those of the infinitesimal one. With SGPC, the performance of the commercial animals is always superior, especially in the case of overdominance or diminishing epistasis. However, as a consequence of matings among relatives induced in the breeding population, the performance of this population was worse when inbreeding depression was present. On the contrary, with SGPC, the inbreeding 
Table III. Performance of the commercial $(R)$ and the breeding population $\left(R^{\prime}\right)$ during five generations of selection using the standard selection procedure and the new method (SGPC) together with the inbreeding coefficient $(F)$. Statistical infinitesimal model. Standard errors of selection responses range between 0.05 and 0.11 .

\begin{tabular}{|c|c|c|c|c|c|c|c|c|c|}
\hline \multirow{3}{*}{\multicolumn{2}{|c|}{ Generations }} & \multicolumn{4}{|c|}{ Commercial population } & \multicolumn{4}{|c|}{ Breeding population } \\
\hline & & \multicolumn{2}{|c|}{$\begin{array}{l}\text { Standard } \\
\text { selection }\end{array}$} & \multicolumn{2}{|c|}{ SGPC } & \multicolumn{2}{|c|}{$\begin{array}{l}\text { Standard } \\
\text { selection }\end{array}$} & \multicolumn{2}{|c|}{ SGPC } \\
\hline & & $R_{S}$ & $F_{S}$ & $R_{N}$ & $F_{N}$ & $R_{S}^{\prime}$ & $F_{S}^{\prime}$ & $R_{N}^{\prime}$ & $F_{N}^{\prime}$ \\
\hline \multirow[t]{7}{*}{ Case } & A & & & & & & & & \\
\hline & 1 & 0.58 & 0.00 & 0.58 & 0.00 & 0.59 & 0.00 & 0.59 & 0.00 \\
\hline & 2 & 1.35 & 0.06 & 1.41 & 0.12 & 1.46 & 0.08 & 1.48 & 0.12 \\
\hline & 3 & 2.19 & 0.11 & 2.39 & 0.22 & 2.23 & 0.12 & 2.40 & 0.21 \\
\hline & 4 & 3.01 & 0.17 & 3.48 & 0.33 & 3.01 & 0.18 & 3.47 & 0.33 \\
\hline & 5 & 3.93 & 0.22 & 4.61 & 0.42 & 3.99 & 0.23 & 4.69 & 0.42 \\
\hline & 6 & 4.86 & 0.27 & 5.85 & 0.49 & 4.87 & 0.29 & 5.82 & 0.47 \\
\hline \multirow[t]{7}{*}{ Case } & B & & & & & & & & \\
\hline & 1 & 0.58 & 0.00 & 0.58 & 0.00 & 0.59 & 0.00 & 0.59 & 0.00 \\
\hline & 2 & 1.23 & 0.06 & 1.32 & 0.03 & 1.30 & 0.08 & 1.23 & 0.12 \\
\hline & 3 & 1.96 & 0.11 & 1.99 & 0.09 & 1.98 & 0.12 & 1.92 & 0.19 \\
\hline & 4 & 2.67 & 0.17 & 2.84 & 0.16 & 2.63 & 0.18 & 2.60 & 0.28 \\
\hline & 5 & 3.47 & 0.22 & 3.73 & 0.23 & 3.51 & 0.23 & 3.54 & 0.36 \\
\hline & 6 & 4.30 & 0.27 & 4.65 & 0.29 & 4.28 & 0.28 & 4.45 & 0.45 \\
\hline \multirow[t]{7}{*}{ Case } & $\mathrm{C}$ & & & & & & & & \\
\hline & 1 & 0.58 & 0.00 & 0.58 & 0.00 & 0.59 & 0.00 & 0.59 & 0.00 \\
\hline & 2 & 1.10 & 0.06 & 1.29 & 0.00 & 1.14 & 0.08 & 0.99 & 0.12 \\
\hline & 3 & 1.73 & 0.11 & 2.00 & 0.03 & 1.72 & 0.12 & 1.52 & 0.20 \\
\hline & 4 & 2.32 & 0.17 & 2.60 & 0.08 & 2.26 & 0.18 & 2.12 & 0.29 \\
\hline & 5 & 3.02 & 0.22 & 3.37 & 0.13 & 3.04 & 0.23 & 2.79 & 0.36 \\
\hline & 6 & 3.74 & 0.27 & 4.17 & 0.18 & 3.70 & 0.28 & 3.52 & 0.44 \\
\hline \multirow[t]{7}{*}{ Case } & $\mathrm{D}$ & & & & & & & & \\
\hline & 1 & 0.58 & 0.00 & 0.58 & 0.00 & 0.59 & 0.00 & 0.59 & 0.00 \\
\hline & 2 & 0.10 & 0.06 & 1.29 & 0.00 & -0.13 & 0.08 & -1.08 & 0.12 \\
\hline & 3 & -0.09 & 0.11 & 2.02 & 0.00 & -0.31 & 0.12 & -2.13 & 0.22 \\
\hline & 4 & -0.46 & 0.17 & 2.59 & 0.00 & -0.73 & 0.18 & -3.29 & 0.31 \\
\hline & 5 & -0.63 & 0.22 & 3.12 & 0.00 & -0.75 & 0.23 & -3.85 & 0.39 \\
\hline & 6 & -0.75 & 0.27 & 3.47 & 0.00 & -0.98 & 0.28 & -4.44 & 0.46 \\
\hline \multirow[t]{7}{*}{ Case } & $\mathrm{E}$ & & & & & & & & \\
\hline & 1 & & & 0.58 & 0.00 & & & 0.59 & 0.00 \\
\hline & 2 & & & 1.26 & 0.00 & & & 1.41 & 0.00 \\
\hline & 3 & & & 1.02 & 0.00 & & & 1.74 & 0.00 \\
\hline & 4 & & & 1.14 & 0.04 & & & 1.58 & 0.04 \\
\hline & 5 & & & 1.21 & 0.07 & & & 1.71 & 0.07 \\
\hline & 6 & & & 1.37 & 0.09 & & & 1.76 & 0.10 \\
\hline
\end{tabular}

Case A: $V_{A}=3.27, V_{D}=6.55, V_{E}=6.55$, no inbreeding depression.

Case B: $V_{A}=3.27, V_{D}=6.55, V_{E}=6.55$, inbreeding depression $=-2.05(F=1)$.

Case C: $V_{A}=3.27, V_{D}=6.55, V_{E}=6.55$, inbreeding depression $=-4.10(F=1)$.

Case D: $V_{A}=3.27, V_{D}=6.55, V_{E}=6.55$, inbreeding depression $=-20.48(F=1)$.

Case E: As case D but with selection for both commercial and breeding population. 
Table IV. Performance of the commercial $(R)$ and the breeding population $\left(R^{\prime}\right)$ after five generations of selection using the standard selection procedure and the new method (SGPC) together with the inbreeding coefficient $(F)$. Model assuming QTLs known. Standard errors of selection responses range between 0.07 and 0.15 .

\begin{tabular}{|c|c|c|c|c|c|c|c|c|c|}
\hline \multirow{3}{*}{\multicolumn{2}{|c|}{ Gen€ }} & \multicolumn{4}{|c|}{ Commercial population } & \multicolumn{4}{|c|}{ Breeding population } \\
\hline & & \multicolumn{2}{|c|}{ Standard selection } & \multicolumn{2}{|c|}{ SGPC } & \multicolumn{2}{|c|}{ Standard selection } & \multicolumn{2}{|c|}{ SGPC } \\
\hline & & $R_{S}$ & $F_{S}$ & $R_{N}$ & $F_{N}$ & $R_{S}^{\prime}$ & $F_{S}^{\prime}$ & $R_{N}^{\prime}$ & $F_{N}^{\prime}$ \\
\hline \multirow[t]{7}{*}{ Case } & $A$ & $(d=0.0)$ & & & & & & & \\
\hline & 1 & 64.90 & 0.00 & 64.90 & 0.00 & 60.03 & 0.00 & 60.03 & 0.00 \\
\hline & 2 & 69.02 & 0.04 & 69.02 & 0.04 & 64.90 & 0.05 & 64.90 & 0.05 \\
\hline & 3 & 72.76 & 0.08 & 72.76 & 0.08 & 68.97 & 0.08 & 68.97 & 0.08 \\
\hline & 4 & 76.23 & 0.12 & 76.23 & 0.12 & 72.75 & 0.12 & 72.75 & 0.12 \\
\hline & 5 & 79.30 & 0.16 & 79.30 & 0.16 & 76.09 & 0.17 & 76.09 & 0.17 \\
\hline & 6 & 82.23 & 0.20 & 82.23 & 0.20 & 79.33 & 0.20 & 79.33 & 0.20 \\
\hline \multirow[t]{7}{*}{ Case } & $B$ & $(d=0.5)$ & & & & & & & \\
\hline & 1 & 79.38 & 0.00 & 79.85 & 0.00 & 76.01 & 0.00 & 76.01 & 0.00 \\
\hline & 2 & 81.51 & 0.04 & 82.37 & 0.00 & 79.38 & 0.04 & 79.11 & 0.09 \\
\hline & 3 & 83.57 & 0.08 & 84.53 & 0.00 & 81.40 & 0.08 & 80.41 & 0.14 \\
\hline & 4 & 85.53 & 0.12 & 86.42 & 0.01 & 83.56 & 0.11 & 82.16 & 0.21 \\
\hline & 5 & 87.41 & 0.15 & 88.05 & 0.02 & 85.57 & 0.14 & 83.54 & 0.27 \\
\hline & 6 & 89.24 & 0.18 & 89.54 & 0.03 & 87.42 & 0.17 & 84.93 & 0.32 \\
\hline \multirow[t]{7}{*}{ Case } & $C$ & $(d=1.0)$ & & & & & & & \\
\hline & 1 & 93.90 & 0.00 & 95.48 & 0.00 & 91.99 & 0.00 & 91.99 & 0.00 \\
\hline & 2 & 94.12 & 0.08 & 96.90 & 0.00 & 93.98 & 0.04 & 92.85 & 0.16 \\
\hline & 3 & 94.73 & 0.11 & 98.12 & 0.00 & 94.14 & 0.07 & 89.23 & 0.26 \\
\hline & 4 & 95.35 & 0.13 & 99.01 & 0.00 & 94.32 & 0.12 & 87.12 & 0.37 \\
\hline & 5 & 95.93 & 0.16 & 99.63 & 0.00 & 95.15 & 0.16 & 84.85 & 0.45 \\
\hline & 6 & 96.43 & 0.19 & 99.92 & 0.00 & 95.81 & 0.19 & 83.08 & 0.51 \\
\hline \multirow[t]{7}{*}{ Case } & $D$ & $(d=1.5)$ & & & & & & & \\
\hline & 1 & 108.59 & 0.00 & 112.09 & 0.00 & 107.97 & 0.00 & 107.97 & 0.00 \\
\hline & 2 & 107.11 & 0.06 & 113.72 & 0.00 & 108.54 & 0.05 & 106.89 & 0.21 \\
\hline & 3 & 106.09 & 0.09 & 115.50 & 0.00 & 106.47 & 0.08 & 96.40 & 0.32 \\
\hline & 4 & 105.38 & 0.12 & 117.12 & 0.00 & 105.81 & 0.11 & 90.70 & 0.43 \\
\hline & 5 & 104.98 & 0.15 & 118.72 & 0.00 & 105.06 & 0.14 & 84.23 & 0.52 \\
\hline & 6 & 104.49 & 0.17 & 120.27 & 0.00 & 104.91 & 0.17 & 78.63 & 0.59 \\
\hline \multirow[t]{7}{*}{ Case } & $F$ & $(d=-1.0$ & & & & & & & \\
\hline & 1 & 35.96 & 0.00 & 36.91 & 0.00 & 28.07 & 0.00 & 28.07 & 0.00 \\
\hline & 2 & 43.94 & 0.04 & 46.88 & 0.18 & 36.01 & 0.05 & 43.94 & 0.15 \\
\hline & 3 & 51.20 & 0.08 & 53.97 & 0.27 & 44.09 & 0.09 & 51.20 & 0.24 \\
\hline & 4 & 57.75 & 0.13 & 60.35 & 0.36 & 51.10 & 0.13 & 57.75 & 0.34 \\
\hline & 5 & 63.81 & 0.18 & 65.41 & 0.43 & 57.98 & 0.18 & 63.81 & 0.42 \\
\hline & 6 & 69.10 & 0.22 & 69.85 & 0.50 & 63.88 & 0.22 & 69.10 & 0.48 \\
\hline \multirow[t]{7}{*}{ Case } & $G$ & $(d=1$ or & $d=-1)$ & & & & & & \\
\hline & 1 & 65.84 & 0.00 & 66.91 & 0.00 & 60.10 & 0.00 & 60.10 & 0.00 \\
\hline & 2 & 70.50 & 0.05 & 71.23 & 0.02 & 65.71 & 0.04 & 65.01 & 0.09 \\
\hline & 3 & 74.56 & 0.08 & 75.51 & 0.04 & 70.42 & 0.08 & 69.25 & 0.14 \\
\hline & 4 & 78.49 & 0.12 & 79.34 & 0.06 & 74.41 & 0.13 & 73.25 & 0.21 \\
\hline & 5 & 82.06 & 0.16 & 82.64 & 0.07 & 78.49 & 0.16 & 76.47 & 0.27 \\
\hline & 6 & 85.09 & 0.19 & 85.73 & 0.08 & 81.81 & 0.21 & 79.06 & 0.32 \\
\hline
\end{tabular}


Table IV. (suite)

\begin{tabular}{cccccccccc}
\hline Case & $H$ & \multicolumn{2}{c}{ (additive $\times$ additive epistasis) } \\
& 1 & 12.16 & 0.00 & 13.56 & 0.00 & 8.01 & 0.00 & 8.01 & 0.00 \\
& 2 & 15.20 & 0.04 & 16.61 & 0.05 & 11.15 & 0.04 & 10.61 & 0.08 \\
& 3 & 18.22 & 0.07 & 19.18 & 0.08 & 13.97 & 0.08 & 13.19 & 0.14 \\
& 4 & 21.16 & 0.12 & 21.96 & 0.13 & 16.79 & 0.11 & 16.23 & 0.18 \\
& 5 & 23.90 & 0.15 & 24.74 & 0.18 & 19.85 & 0.14 & 19.17 & 0.24 \\
& 6 & 27.04 & 0.20 & 27.83 & 0.23 & 22.90 & 0.17 & 22.46 & 0.29 \\
Case & $I$ & \multicolumn{2}{c}{ (synergistic epistasis) } & & & & & \\
& 1 & 122.10 & 0.00 & 123.09 & 0.00 & 115.58 & 0.00 & 115.58 & 0.00 \\
& 2 & 126.26 & 0.04 & 128.41 & 0.00 & 122.30 & 0.05 & 121.30 & 0.10 \\
& 3 & 129.96 & 0.07 & 133.19 & 0.00 & 125.91 & 0.08 & 122.85 & 0.17 \\
& 4 & 133.21 & 0.11 & 137.65 & 0.00 & 129.93 & 0.11 & 124.80 & 0.25 \\
& 5 & 136.81 & 0.13 & 141.67 & 0.01 & 133.43 & 0.14 & 125.85 & 0.31 \\
& 6 & 140.07 & 0.16 & 145.57 & 0.01 & 136.70 & 0.17 & 126.91 & 0.37 \\
\hline
\end{tabular}

of commercial population is lower than with the standard method, and in the cases of complete dominance and overdominance, the avoidance of inbreeding is maximum.

However, in the case of $d=-1$ the SGPC induces inbreeding in both the commercial and the breeding population, because in this case inbreeding increases the genetic mean and therefore both populations have better performance than with the standard method. And if inbreeding depression is absent, as in the case of additive $\times$ additive epistasis [2] or when positive and negative effects of inbreeding are cancelled because at half of the loci $d=1$ and at the other half $d=-1$ (case G, table $I V$ ), the optimal level of inbreeding is automatically adjusted.

\section{DISCUSSION}

Although the idea of using deliberate inbreeding in selection programmes is generally disfavoured in animal breeding, several authors have indicated that a reappraisal of the subject is needed [5, 12]. Inbreeding has two opposite effects. It increases selection response because it allows the accumulation of dominance effects but it also decreases genetic mean due to inbreeding depression. The SGPC method proposed here is intended to take simultaneously into account both aspects of the problem. The idea is that we should select grandparental combinations such that the overall genetic merit of future commercial grandoffspring will be maximum. In this way, the proportion of matings among relatives is optimized both to obtain commercial animals and to propagate the population.

The main aim of the present paper has been to propose this new procedure, which appears to be a general method of utilizing additive and dominance effects. The method has been checked by computer simulation of a breeding scheme which was unrealistically small in order to achieve computational feasibility and assumed an unrealistically high value of the dominance variance (twice the additive variance) in order to magnify the difference between the methods. Despite this large assumed variance, the improvement was less than 
$15 \%$ in cases $\mathrm{B}$ and $\mathrm{C}$ (table $I I I)$. This casts some doubt on the practical advantages of the new method and more work remains to be carried out simulating more practical situations of current nuclei of selection including the cost associated with inbreeding depression of the breeding population and specifying the structure of dissemination of genetic progress. But two facts should be kept in mind. First, recent developments have allowed computations with models including dominance [10]: this has created the possibility of obtaining a benefit from such evaluation even if it is small. Second, the method could also be generalized to include multibreed situations. In crossbreeding the method will optimize the matings to be made in pure breeds in order to achieve maximum profit from commercial crossbred grandoffspring.

The new method has some analogies with reciprocal-recurrent selection. Both methods rely on the crucial distinction between commercial and breeding populations. But RRS begins with two populations, and an essential prerequisite is that there should be some difference in gene frequency between the two lines at the beginning [1]. The start of SGPC is a closed population and any subsequent subdivision that can occur in the breeding population will be a consequence of the selection process and will depend on the genetic basis of the selected trait.

Some theoretical and estimation problems remain if additional phenotypic information is used. In the present paper evaluation is based only on information coming from the nucleus but it could be improved if information from commercial animals of previous generations might be included. We have also used a straightforward infinitesimal model that includes dominance variance and that accounts for the average effect of inbreeding on the mean by including the inbreeding coefficient as a covariate. The value of this approach has been discussed by de Boer and van Arendonk [3], but it is clear that for a detailed understanding of how the SGPC method is working a more sophisticated model for simulating and analysing the data is needed. The best candidate is the model proposed by Smith and Maki-Tanila [11], which considers the reduction of base dominance variance, the increase in dominance variance of completely inbred individuals and the covariance among additive and dominance effects with inbreeding.

The present study has additional limitations requiring further research. The properties of SGPC in the medium and long term have not been investigated but it can be conjectured that the additive variance in the long term will be reduced, since the method imposes some inbreeding in the breeding population. Furthermore, computation could also be a limiting factor: with $\mathrm{N}$ grandparents of each sex, there will be $\mathrm{N}^{4}$ grandparental combinations. The present study has shown that dominance genetic effects can be accumulated by adequate planning of selection and mating policy.

\section{REFERENCES}

[1] Comstock R.E., Robinson H.R., Harvey P.B., A breeding procedure designed to make maximum use of both general and specific combining ability, Agronomy J. 41 (1949) 360-367.

[2] Crow J.F., Kimura M., An introduction to population genetics theory, Harper $\&$ Row, New York, 1970. 
[3] De Boer I.J.M., Van Arendok J.A.M., Prediction of additive and dominance effects in selected and unselected populations with inbreeding, Theor. Appl. Genet. 84 (1992) 451-459.

[4] DeStefano A.L., Hoeschele I., Value of including dominance genetic merit in mating progress, J. Dairy Sci. 75 (1992) 1680-1690.

[5] Dickerson G.E., Lindhé N.B.H., Inbreeding and heterosis in animals, in: Pollack E., Kempthorne O., Bailey Jr. (Eds.), Proceedings of the International Conference on Quantitative Genetics, Iowa State University Press, 1977, pp. 323-342.

[6] Henderson C.R., Best linear unbiased prediction of nonadditive genetic merit in mating programs, J. Dairy Sci. 84 (1985) 451-459.

[7]. Hoeschele I., Van Raden P.M., Rapid inversion of dominance relationship matrices for noninbred populations by including sire $\mathrm{x}$ dam subclass effects, J. Dairy Sci. 74 (1991) 557-569.

[8] Jansen G.B., Wilton J.W., Selecting mating pairs with linear programming techniques, J. Dairy Sci. 68 (1985) 1302-1305.

[9] Kinghorn B., On computing strategies for mate allocation, J. Anim. Breed. Genet. 104 (1987) 12-22.

[10] Misztal I., Lawlor T.J., Gengler N., Relationships among estimates of inbreeding depression, dominance and additive variance for linear traits in Holsteins, Genet. Sel. Evol. 29 (1997) 319-326.

[11] Smith S.P., Maki-Tanila A., Genotypic covariance matrices and their inverses for models allowing dominance and inbreeding, Genet. Sel. Evol. 22 (1990) 65-91.

[12] Toro M., A new method aimed at using the dominance variance in closed breeding populations, Genet. Sel. Evol. 25 (1993) 63-74.

[13] Toro M.A., The utilization of dominance genetic effects in selection schemes, in: Proceedings of the 5th World Congress on Genetics Applied to Livestock Production, Guelph, 7-12 August 1994, University of Guelph, Canada, 1994, 262-65.

[14] Wei M., Van der Steen H.A.M., Comparison of reciprocal recurrent selection with pure-line selection systems in animal breeding (a review), Anim. Breed. Abstr. 59 (1991) 281-298. 